\title{
AAPS Workshop on the Role of Dissolution in QbD and Drug Product Life Cycle: A Commentary
}

\author{
Susan S. D'Souza ${ }^{1, *}$ Ruben Lozano², Stephen Mayock ${ }^{3}$, and Vivian Gray ${ }^{4}$ \\ 'Chemistry and Pharmaceutical Sciences, Sunovion, Inc., Marlborough, MA, USA \\ ${ }^{2}$ Analytical Research \& Development, Bristol-Myers Squibb, New Brunswick, NJ, USA \\ ${ }^{3}$ Vertex Pharmaceuticals, Inc., Cambridge, MA, USA \\ ${ }^{4}$ V. A. Gray Consulting, Inc., Hockessin, DE, USA
}

\begin{abstract}
This is a summary report of the "AAPS Workshop on the Role of Dissolution in QbD and Drug Product Life Cycle" organized by the AAPS In Vitro Release and Dissolution Testing (IVDRT) Focus Group. Representatives from the pharmaceutical industry, regulatory authorities, and academia in the U.S. and Europe attended this workshop to discuss the role of dissolution in a Quality by Design $(\mathrm{QbD})$ setting and its relevance in drug product development. Other areas of discussion included IVIVC/R and hot topics like alcohol dose-dumping. Numerous case studies were presented, and issues relevant to the dissolution scientist and areas needing further research were highlighted at this workshop. Views expressed in this paper are those of the participants from the industry and the agency and do not necessarily represent those of the FDA and USP.
\end{abstract}

\section{INTRODUCTION}

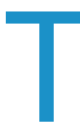
his paper summarizes the panel discussions of the "AAPS Workshop on the Role of Dissolution in QbD and Drug Product Life Cycle" held on April 28-30, 2008 in Crystal City, Va., and jointly sponsored by the American Association of Pharmaceutical Scientists (AAPS) and the U.S. Food and Drug Administration (FDA) (1). The workshop was a follow-up to a previous meeting (2) organized by the AAPS In Vitro Release and Dissolution Testing (IVDRT) Focus Group, and highlights have been published (3). This two-and-a-half-day workshop provided a forum for participants and experts from the pharmaceutical industry, the regulatory authorities, and academia in the United States and Europe to share current thinking on the role of dissolution testing in the following areas:

- Role of Dissolution in QbD and Product Development Continuum (Session 1)

- Role of Dissolution in QbD (Session 2)

- Relevance of Dissolution in Drug Development (Session 3)

- IVIVC/R (Session 4)

- Dissolution: Hot Topics (Session 5)

Each session consisted of a series of presentations followed by a Q\&A session. A panel discussion was conducted at the end of each session. This summary paper is based on the panel discussions and is divided into five sections, one for each session.

*Corresponding author.

\section{SESSION 1: ROLE OF DISSOLUTION IN QBD AND PRODUCT DEVELOPMENT CONTINUUM}

The panel discussion centered around three points. The first topic was the importance of having the in vitro test reflect bioequivalence and further the understanding of the release mechanism. In such cases, the BCS classification should be taken into account so that product quality can be related to clinical relevance. In some cases, as with BCS Class 1, in vitro studies could be more appropriate than in vivo testing in terms of total cost and may actually better assess the performance of the product. It is necessary for the formulator to be more critical of the formulation and to understand the release mechanisms, especially when there is high in vivo and in vitro variability. The intrinsic variability can be high when there is a firstpass effect combined with low solubility and when the $\mathrm{p} K_{\mathrm{a}}$ is close to the gastric $\mathrm{pH}$. Sometimes the in vivo result can be inaccurate, showing that the BCS class needs to be considered when relating quality to clinical relevance.

Another point of discussion centered on the difficulty in applying QbD principles to in vitro testing given the limited time available for development. This point was challenged, especially with regard to generic products where prior knowledge of the formulation may be available. An understanding of the release mechanism of the generic formulation is important since in many cases there are different excipients that can pose comparable change in the release mechanism. All agreed that the safety of the product should always prevail. There was discussion regarding use of the peak vessel, since it is not favored by the FDA but has been used in a few approved 
products. Because it is not standard equipment, peak vessel use requires documented justification. It was pointed out that increasing paddle speed often eliminates the cone, but there are limits as to how high the paddle speed can go without losing discriminatory power. Though a comment was made that reducing the amount of excipients in the formulation could reduce or eliminate the cone, it was pointed out that cone formation and dissolution should not be the driving force to modify a formulation. It was noted, however, that robustness is an issue with the official round-bottom vessel, and this would carry over with the peak vessel.

It was noted by the FDA representative that by using QbD principles (i.e., having a well-designed space), one could eliminate dissolution altogether. For example, if particle size correlates with dissolution during development, then particle size measurement could be used in lieu of the latter. The agency is trying to encourage multinational companies to use the QbD approach, but this is not easy unless it is adopted globally.

Related to QbD, the BCS classification was mentioned, and most agreed that BCS Class 1 rarely, if ever, failed an in vivo study; if Class 3 failed, it was unusually underpowered. There was a prevailing view by the audience that Class 2 compounds, with appropriate media, could be good candidates for a biowaiver.

\section{SESSION 2: ROLE OF DISSOLUTION IN QBD}

Panel feedback was requested for a scenario involving an in vitro-in vivo correlation (IVIVR) in which API particle size was a critical variable. Since development of the $\mathrm{QbD}$ was to be based upon this IVIVR, would it be acceptable to use IVIVR to map out the design space? The response to this question was that it is difficult to assess this without knowing more about the properties of the compound and whether the API particle size is the highest risk output. If the product is a simple formulation and API particle size is the critical variable having a dominant effect on the dissolution rate, then this approach might be acceptable. The FDA representative agreed.

With the advent of $\mathrm{QbD}$, dissolution may not be required as a product release specification. In fact, there are cases where disintegration is used as a surrogate test for a few approved products. However, it is important to note that dissolution is one of the most important quality attributes for QbD studies. Sometimes there may be no significant differences in dissolution results across the design space. In such cases where the dissolution method does not appear to be discriminating, a good starting point would be to set up the design space with all the parameters that might influence or interact with one other and impact dissolution results. The next step is to determine the parameters that are critical and identify those that are clinically relevant. Prior knowledge of the product is also helpful. For example, if particle size does not influence dissolution, as is the case with BCS Class 1 or 3 drugs, then this result is expected. However, if the drug belongs to Class 2 or 4, then the dissolution method should be revisited. The importance of performing dissolution experiments in the early stages of drug development cannot be emphasized enough. A properly designed DOE followed by statistical analysis is required to gain a thorough understanding of the physical properties of the drug product, API, and excipients. In a QbD world, dissolution is not a standalone test. It should be recognized that dissolution results are relevant not only to the analyst, but also to the formulator and the clinician. Hence, it is important to understand the formulation as well as the release mechanism of the drug product.

When QbD principles are used to establish dissolution specifications, identification of the Critical Quality Attributes (CQA) should be done, followed by risk assessment, process analysis, and finally, determination of design space. The final goal of $\mathrm{QbD}$ is to develop meaningful specifications, and this approach utilizes the relationship that exists between CQAs and specifications. From a regulatory standpoint, SUPAC has been used by the agency as a risk-assessment tool for different types of products. However, in a QbD environment where the manufacturer has intimate knowledge of the product, design space is expected to be larger than the typical operating ranges of products that have been approved in the past, and hence, SUPAC may not apply to genuine QbD applications.

Traditionally, the general rule of thumb for specifications used to monitor batch-to-batch variation in immediate-release products is $\pm 10 \%$ or \pm 2 or 3 standard deviations. This is also true for controlled- and extendedrelease products. However, this may or may not relate well with in vivo data. When dealing with controlled- or extended-release products, it is important to bear in mind that the dissolution specification should have some clinical relevance. There has to be a degree of correlation between the in vitro test and in vivo product performance. When viewing dissolution as a key to simulating in vivo performance, establishing proper specifications becomes even more important if IVIVC is the ultimate goal. An example of a marketed controlled-release product was cited to illustrate this point. For this product, new API suppliers were being considered, and as part of the evaluation process, dissolution tests were performed on the product containing API from the new suppliers. Because the specifications were wide, API from all the new suppliers appeared to meet the criteria. From prior knowledge, the thought was that the final product could be bioinequivalent, and hence, a decision was made to initiate a large clinical trial in humans using multiple formulations with the goal of establishing an IVIVC. These data were used to redefine and tighten the dissolution specification.

During the panel discussion, clarification was sought regarding the need for a dissolution method to be biorelevant or useful in establishing IVIVC, given that most products do not use biorelevant methods. To this point, one panelist stated that most dissolution methods are 
developed during Phase I where the specifications are typically wide. Once a method has been developed and specifications established, little or no change is made to the dissolution method. If $\mathrm{QbD}$ is the goal, it is important to revisit the method so that it is discriminatory in nature and also has some clinical relevance. This will build a measure of trust and quality into the product.

The consensus of the panelists from the agency was that the dissolution test should not only be used as a quality control measure for batch-to-batch release, but that it could be made more physiologically relevant, which would benefit the patient. The FDA panelists also noted that their European counterparts were ahead in their thinking in this regard. One panelist suggested that a flowchart be adopted to enable the decision-making process.

\section{SESSION 3: RELEVANCE OF DISSOLUTION IN DRUG DEVELOPMENT}

Panel discussion for the third session focused on three topics. One topic was TNO-TIM food-effect modeling that has recently gained some attention. An example of TIM modeling was cited for a tablet formulation where various media (FASSIF, FESSIF, SGF, ground-up meal) were applied. It was also noted that there are several published examples of food-effect modeling using TNO-TIM with nutraceuticals to better understand food digestion. Some audience members who have used TNO-TIM commented that much preliminary work has already been done to understand and establish setup parameters. The general feeling was that TNO-TIM modeling would be of most value in latestage development.

Another topic of discussion centered on solubility. A comment was made that it is difficult to predict or calculate solubility of different salt forms based simply on $\mathrm{p} K_{\mathrm{a}}$ and intrinsic solubility data, because salt solubility is not solely dependent on the $\mathrm{pH}$ of the medium. Other variables such as hydrogen bonding, ionic bonding, and lattice-structure effects may contribute to the solubility, and some of these effects have been discussed in a review article by Serajuddin (4). One approach to better understand solubility would be to investigate in situ salt solubility at an early stage using a small amount of drug to differentiate solubility driven by $\mathrm{pH}$ versus other contributing factors. For studies to screen drug dispersions, the recommended drug-to-polymer surfactant ratio should start at 10:90, then be scaled down or up to find the ideal solubilization.

The last discussion point for the third session focused on osmolality measurement when using the two-stage dissolution method (SGF followed by FASSIF). There was a concern that although the end mixture may have the same $\mathrm{pH}$ and surface tension as FASSIF, it likely has an osmolality different from true gastric fluid, whereas FASSIF may mimic the Gl tract more closely. A truly biorelevant medium should therefore mimic physiological conditions as closely as possible including all the physicochemical properties.

\section{SESSION 4: IVIVC/R}

Panel discussion began with the topic of biowaivers for BCS Class 1 drugs, for which the standard dissolution test conditions stipulate a paddle rotation speed of $50 \mathrm{rpm}$. However, for certain formulations that exhibit coning during dissolution, a paddle speed of $75 \mathrm{rpm}$ is generally accepted. The FDA representative replied that the reviewing committees realize the justification for the higher 75-rpm paddle speed in special cases, and they would consider acceptance of its implementation in biowaiver requests for products that demonstrate coning. On a related note, some BCS 2 drugs that are poorly soluble weak bases can have sufficient solubility and bioavailability in the lower GI tract and therefore might be considered by some as "borderline BCS 1" for purposes of biowaiver requests. Although weak acids are more likely to dissolve better in the lower Gl tract, some weak bases may precipitate and not be eligible for BCS 1 biowaiver. For BCS 1 prodrug products whose bioavailability is $90 \%$ of reference, any decisions by regulatory agencies about a dissolution-based biowaiver must be made on a case-by-case basis. More data may be needed about the specific prodrug (e.g., primary site of metabolism). If bioavailability is $90 \%$, absorption is also likely to be high (90\%), then high permeability should be demonstrated or mass balance studies performed.

The next topic of discussion was the regulatory acceptance of real-time release and the elimination of dissolution as a release test. In one of the case studies presented, HPMC level was a critical attribute, but other tests for monitoring batch consistency were also investigated using the elements of design space. For example, granule size was monitored although it was deemed not critical, but data were accumulated to ensure that all parts of the process were working properly. Granule size could perhaps be used as a future surrogate test for particle size distribution. Although USP specifications are in place for all the excipients and the supplier has internal specifications based on the USP specs, there is still a need to characterize excipient variability, which can be done using a QbD approach. If critical process parameters (CPP) and critical material attributes (CMA) are understood and controlled, then dissolution may not be needed for release. However, dissolution testing still has value, such as for biowaivers, in determining the rate and amount of drug released.

The next item on the panel discussion was the demonstration of IVIVC for BCS 2 drugs, which can be very difficult and perhaps improbable in many cases. The FDA representative acknowledged that the agency may accept IVIVR data for biowaivers in the future. Indeed, the agency will consider any science-based justification. Although it is difficult to predict BCS 2 in vivo performance, it is hoped that useful in vitro methods can still be developed. Although additional work in performing in vitro studies may be needed, the result can be favorable due to a reduction in the number of in vivo studies. 
On the topic of biorelevance, it was noted that there have been multiple interpretations of this often-used term. It was agreed that two separate definitions of a biorelevant medium need to be established: (1) a medium that mimics the physiological environment of some part of the $\mathrm{Gl}$ tract, and (2) a medium resulting in a dissolution test that demonstrates some relevance to clinical performance of the drug product. A remark was made that it would be convenient if bile salts in the traditional biorelevant media could be replaced with some other component (e.g., a common surfactant) for purposes of a simpler, less expensive medium. However, selection of appropriate surfactants as bile salt replacements is difficult because their performance can be unpredictable in the biorelevant media due to the effects when mixed with excipients. It is desirable to have a flow chart for surfactant use in dissolution media. It would also be convenient to have a dissolution test consisting of standardized instrumentation and medium that would make it suitable for determination of biorelevance yet not as complex as methods employing traditional biorelevant media. However, simple media cannot be easily employed during early development because of the need to investigate first the addition of different types of surfactants.

A few questions related to permeability studies, simulation software, and the focus of dissolution tests were also posed to the panelists. Clarification was sought on whether permeability studies should be conducted on the highest strength of the product or on the highest dosage (which may consist of multiple dosage forms of the highest strength). The reply was that the actual dose being studied in the clinic should be the basis of the permeability study. Regarding simulation software, it was observed that conclusions may sometimes be misleading. To ensure that one arrives at the proper conclusion, the panelist recommended that the input values be more accurate. The panelist also commented that the software has proved useful in early stages by providing general direction for formulation development. Although additional in vitro and in vivo studies may be needed, they may be greatly reduced as a result of the simulations. Not every simulation is meaningful, thus more input is generally necessary. Another question was asked as to whether the dissolution test should be generic or more product-specific. The response was that a mechanistic approach should be taken, based on the objective of the test, whether it be a method for quality control or batch consistency (simple approach) or a method for biorelevance (more complex approach). The volume of the dissolution medium may have biorelevant impact. If the method purpose is to mimic the GI tract, then sink conditions should be the main consideration in volume selection. It is difficult to simulate the Gl tract exactly, but generally smaller volumes such as $500 \mathrm{~mL}$ are used for biomimicking-type media. Often, larger volumes of 900 or
$1000 \mathrm{~mL}$ are used to accommodate future formulation changes (e.g., higher strengths).

\section{SESSION 5: DISSOLUTION: HOT TOPICS}

The first topic of the panel discussion focused on alcohol dose-dumping. When an immediate-release formulation of a drug with abuse potential (e.g., hydromorphone) is ingested with alcohol, its permeability may be enhanced, leading to toxic levels in the blood. A similar situation exists for drugs with narrow therapeutic indexes, where alcohol consumption could cause fatal side-effects. For such situations, one of the questions raised was whether the indications (for administration) could be revised keeping the side-effects in mind. The panelists responded that in their experience, slight increases in permeability have not translated into dosedumping events. Also, no fatal side-effects have been observed so far with these drugs, and hence, a blanket statement correlating the increase in permeability with dose-dumping is incorrect. However, there is a potential for dose-dumping to occur, and the panelists felt that it should be assessed by clinicians and post-marketing on a case-by-case basis. Overall, the panelists agreed that from a clinician's perspective, dose-dumping has an unacceptable risk-benefit ratio.

A concern was raised on the use of high levels of alcohol in the dissolution media during in vitro testing for alcohol dose-dumping. Due to the increased potential for flammability in an open, heated dissolution bath, the level of alcohol may be a safety concern. The agency responded by suggesting that one can certainly be clever about designing the apparatus. However, it is better to use a QbD approach for dosage-form design, wherein one can predict what kind of dosage form would cause dose-dumping, or work toward forming a matrix system to avoid this phenomenon. In addition, the agency reminded the audience that the principal consideration in alcohol dose-dumping is in vivo, not in vitro, performance.

A patent-law question focused on the award of a patent in light of an identical biopharmaceutical process given that crystals or co-crystals are accompanied by a therapeutic claim or biopharmaceutical process that could be equivalent. The panelist responded that if one can assume that the compound still carries its therapeutic use and efficacy, it could be used to fulfill the utility requirement in the patent application. If a compound has different crystal structures that show differences during dissolution (unique to crystal form), but therapeutic use and in vivo behavior are the same, a patent may be issued based on the crystal properties. The approach for patenting co-crystals should be similar to that for crystals and should meet utility requirements.

Another comment from the audience addressed by the agency was on the use of draft guidances that are available online or withdrawn. The agency representative felt that draft guidances that have been published or finalized and are currently available online (but not 
withdrawn) are considered to be appropriate. Previous guidances could represent a starting point because at one time, they reflected the agency's viewpoint.

On the topic of mechanical calibration, it was commented that the agency field investigators were not trained in this aspect per the USP guidelines. The agency representative replied that work was being done to improve this effort. Additionally, the agency representative stated that all guidances are nonbinding and may be accepted by either party as a reasonable approach or as a starting point. Nonconformance with what is expressed in the guidance may or may not result in a 483.

In responding to the relevance of limits $( \pm 40 \%)$ when using Apparatus 2, given the range of the specifications, the expert committee representative stated that the limits are not a standard and could be made tighter, but more research is needed to answer this question. The expert committee is looking into this. On the same subject, a comment was made on the tolerances in the $<711>$ toolkit. These have been tightened, and this will impact the harmonized chapter. The USP representative replied that there are plans to appraise the EP and JP and keep the chapter harmonized.

The audience posed a question about a combination product where a patent was filed based on the analytical method. An NDA was obtained; however, the patent was challenged and then rescinded, by which time the product patent had expired. In such situations, could analytical methods be used to protect intellectual property (from a legal standpoint), or if not, could the dissolution method that is fully correlated with pharmacokinetic data be used to protect intellectual property? The agency representative replied that the agency does not evaluate the merits of the patent cited; it simply requires patent certification provided in an application. It is between the patent holder and other parties to address merits of the patent or lack thereof. The patent attorney said that you can get a patent on any invention that is useful, including an analytical method. Whether the patent becomes a standard is a separate issue because of royalties to be paid. The panelists agreed that that for a combination product having established safety and efficacy for individual components, additional analyses have to be performed to evaluate if previously used methods are still applicable.

A member of the audience commented that models for comparing disintegration and dissolution are inappropriate, especially when the dissolution profile is analyzed to assess whether disintegration is the rate-limiting step. Also, it is inappropriate to compare disintegration time, which is the completeness of the process, with the $Q$ value, which is one parameter of an entire profile. One should bear in mind that that the dissolution profile should be scrutinized, and proof should be provided that the $Q$ value is disintegration plus deaggregation at one time. The panelist responded that one must attempt to correlate disintegration and dissolution events. Another panelist added that there is a provision that disintegration can be used as a surrogate for dissolution for a few products (on a case-by-case basis), provided mechanisms are well understood. Additionally, an audience member noted that one has to be aware of the differences in beaker size between USP $<701>$ and $<2040>$ (dietary supplements) if the disintegration test will be used as a surrogate for dissolution.

Another comment regarding the BCS classification was that it should be based on in vivo data. There is a possibility that permeability may be underestimated under in vitro conditions. Therefore, based on in vivo results, a Class 4 drug (poor permeability) may be classified as Class 2 (high permeability).

Finally, the panel discussion closed with a comment that disintegration and dissolution are important tests that should be measured at the same time point. Every effort should be made to use the QbD approach for drug products, including fixed-dose combinations.

\section{REFERENCES}

1. 2008 AAPS Dissolution Workshop Planning Committee:Tahseen Mirza, Ph.D., Novartis Pharmaceutical Corporation, Co-chair; Helen Winkle, U.S. Food and Drug Administration, Co-chair; William Brown, U.S. Pharmacopeia; Susan D'Souza, Ph.D., Sepracor, Inc.; Vivian Gray, V.A. Gray Consulting, Inc.; Mansoor Khan, Ph.D., U.S. Food and Drug Administration; John Kovaleski, Ph.D., Teva Pharmaceuticals; Ruben Lozano, Ph.D., Bristol-Myers Squibb Company; Stephen Mayock, Catalent Pharma Solutions; Moheb Nasr, Ph.D., U.S. Food and Drug Administration; Alger Salt, GlaxoSmithKline; John Smith, Ph.D., U.S. Food and Drug Administration; Kailas Thakker, Ph.D., Analytical Solutions, Inc.; Saji Thomas, Par Pharmaceuticals; Cheng Tong, Ph.D., Pfizer, Inc.; Qingxi Wang, Ph.D., Merck and Company, Inc.; Lawrence Yu, Ph.D., U.S. Food and Drug Administration; Melvin H.Weinswig, Ph.D., University of WisconsinMadison, Continuing Education.

2. Tong, C.; D'Souza, S. S.; Parker, J. E.; Mirza, T. Commentary on AAPS Workshop: Dissolution Testing for the Twentyfirst Century: Linking Critical Quality Attributes and Critical Process Parameters to Clinically Relevant Dissolution. Pharm. Res. 2007, 24 (9), 1603-1607.

3 Thakker, K.; Gray, V. Meeting Report: AAPS Workshop on the Role of Dissolution in QbD and Drug Product Life Cycle. Dissolution Technol. 2008, 15 (3), 39-41.

4. Serajuddin, A.T. Salt formation to improve drug solubility. Adv. Drug Deliv. Rev. 2007, 59 (7), 603-616. 